\title{
Abnormal expression of Forkhead Box J2 (FOXJ2) suppresses migration and invasion in extrahepatic cholangiocarcinoma and is associated with prognosis
}

\author{
YONG QIANG $^{1,2^{*}}$, FEIRAN WANG $^{1 *}$, SUJUAN YAN $^{3}$, HAITAO ZHANG $^{4}$, LIRONG ZHU $^{1}$, ZHEN CHEN $^{1}$, \\ FANG TU $^{5}$, DONGZHI WANG ${ }^{1}$, GANG WANG $^{1}$, WEI WANG ${ }^{1}$ and ZHONG CHEN $^{1}$ \\ ${ }^{1}$ Department of Hepatobiliary Surgery, Affiliated Hospital of Nantong University and \\ Research Institute of Hepatobiliary Surgery of Nantong University, Nantong, \\ Jiangsu 226001; Departments of ${ }^{2}$ General Surgery and ${ }^{3}$ Gynaecology and Obstetrics, \\ The First People's Hospital of Jingmen, Dongbao District, Jingmen, Hubei 448000; \\ ${ }^{4}$ Jiangsu Key Laboratory of Neuroregeneration, Nantong University, Nantong, Jiangsu 226001; \\ ${ }^{5}$ Department of Operating Rooms, Shayang People's Hospital, Shayang, Hubei 448200, P.R. China
}

Received February 2, 2015; Accepted March 30, 2015

DOI: $10.3892 /$ ijo.2015.2957

\begin{abstract}
Extrahepatic cholangiocarcinoma (CC) is an aggressive malignancy with dismal prognosis and characterized by early invasion, metastasis and postoperative recurrence. Therefore, understanding the main molecular mechanisms of this malignancy is the key for the development of novel and effective therapeutic strategies for extrahepatic CC. Foxj2 is a novel forkhead factor. Several FOX family members have been reported to play an important role in tumorigenesis and the progression of certain cancers. In this study, realtime quantitative RT-PCR (qRT-PCR), western blotting, and immunohistochemical staining were used to examine FOXJ2 expression in extrahepatic $\mathrm{CC}$ tissues and adjacent normal bile duct tissues. The molecular mechanisms of FOXJ2 expression and its effects on cell proliferation, migration and invasion were also explored by MTT assay, wound healing assay and Transwell assay. The relationships between the FOXJ2 expression levels, the clinicopathological factors, and patient survival were investigated. FOXJ2 mRNA and protein levels were downregulated in extrahepatic CC tissues compared to adjacent normal bile duct tissues. In addition, decreased FOXJ2 was associated disease progression in extrahepatic CC
\end{abstract}

Correspondence to: Professor Zhong Chen, Department of Hepatobiliary Surgery, Affiliated Hospital of Nantong University and Research Institute of Hepatobiliary Surgery of Nantong University, 20 Xisi Road, Nantong, Jiangsu 226001, P.R. China

E-mail: ntfychenz9806@163.com

*Contributed equally

Key words: extrahepatic cholangiocarcinoma, FOXJ2, prognosis, epithelial-mesenchymal transition samples. Overexpression FOXJ2 expression markedly inhibited cell proliferation, migration and invasion in vitro. FOXJ2 is a transcription factor that has been reported to induce epithelialmesenchymal transition (EMT). These findings indicated that FOXJ2 gene played a tumor suppressor role in extrahepatic $\mathrm{CC}$, which proposed this gene as a new therapeutic target for extrahepatic CC patients.

\section{Introduction}

Cholangiocarcinoma (CC) is a malignant tumor that arises from the ductal epithelium of the biliary tree (1). CC accounts for $\sim 3 \%$ of all gastrointestinal malignancies; however, it represents the second most common hepatic malignancy after hepatocellular carcinoma (HCC) (2). CC is currently classified as intrahepatic $\mathrm{CC}$ and extrahepatic $\mathrm{CC}$, depending on the carcinogenic site. Extrahepatic CCs are further divided into hilar (Klatskin tumor) or distal tumors and the incidence of this cancer has been increasing in recent years (3). Surgical resection remains the only potentially curative therapeutic option, however, as a result of the early invasion and metastasis, more than half of patients present with unresectable disease at diagnosis (4). Although many patients may receive extensive surgical resection and adjuvant chemotherapy to improve chance of cure, the therapeutic effect and postoperative prognosis are still unsatisfactory, with a 5-year survival rate of $30-42 \%$ for hilar CC, and $18-54 \%$ for distal CC $(5,6)$. Thus, discovery of new relevant biomarker to increase specificity or sensitivity for early diagnosis and to improve the prognosis of extrahepatic CC is important and urgently needed.

Fork-head factors are transcription factors that share an evolutionarily conserved DNA-binding domain, termed the 'fork-head' or 'winged-helix' domain (7). Most members of the Fork-head Box (FOX) family have been reported to be widely distributed across several organs and tissues in very different species from yeast to humans and play extraordinarily diverse 
roles that are critical to the organism (8). Nowadays, mounting evidence suggests that FOX family members are important for a wide spectrum of biological processes, including metabolism, development, differentiation, proliferation, apoptosis, migration, invasion, and longevity (9). Deregulation of FOX family genes leads to congenital, diabetes mellitus, or carcinogenesis (10).

Given their important role in the expression of numerous genes that affect cell proliferation, differentiation and survival, FOX family members may represent direct targets, and indirect effectors of therapeutic intervention. In addition, FOX family members have been shown to be upregulated or downregulated in many cancers. For example, Foxp1 transcription factor may function as either an oncogene or as a tumor suppressor depending on the cell types (11-14). FOXA1 gene is amplified and overexpressed in esophageal and lung cancer (15). Furthermore, FOXJ1 was remarkably upregulated in human HCC specimens (16). Increased expression of FOXM1 protein was found in variety of human tumors, including HCC (17), intrahepatic CC (18).

Foxj2 is a novel forkhead factor, belonging to the FOX family, with a dual DNA binding specificity (19). Some studies have shown that FoxJ2 is a transcription factor of this family that shows a rather broad expression pattern, both in the adult as well as during embryonic development, but the levels of expression vary between organs and, in each organ, not all cells types express the factor (20). Recent study has reported that upregulation of FOXJ2 might inhibit of cell migration and invasion of breast cancer (21). However, the role of FOXJ2 in EHCC has not been explored thus far. In this study, we surveyed the expression of FOXJ2 in human patient samples. To explore its associated molecular mechanisms in extrahepatic CC cells, we examined the effect of targeted overexpression of FOXJ2 gene on cell proliferation, migration and invasion in vitro. These studies will be useful in identifying potential candidates for targeted therapeutic intervention of extrahepatic CC.

\section{Materials and methods}

Patients and clinical samples. We included a total of 63 paraffin-embedded extrahepatic $\mathrm{CC}$ and matched paracancer normal bile duct tissue samples from patients who underwent surgical treatment at Affiliated Hospital of Nantong University during the period from January 2005 to January 2009. The patients or their legal guardian provided written informed consent to the surgical procedures and gave permission to use resected tissue specimens for research purposes. The patients with preoperative history of radiotherapy, chemotherapy, and positive surgical margins were excluded. Furthermore, a diagnosis of extrahepatic CC was confirmed pathologically by two independent experienced pathologists. All specimens excluding the pathological diagnosis of pancreatic ductal carcinoma and periampullary carcinoma confirmed pathological diagnosis and were classified according to the World Health Organization (WHO) criteria. The tumor stage was performed according to the 7th Union for International Cancer Control (UICC)-TNM Staging. The follow-up data of the extrahepatic CC patients in this study are available and complete. Overall survival, which was defined as the time from the operation to the time of patient death or the last follow-up, was used as a measure of prognosis. Postoperative follow-up occurred at our outpatient department and included clinical and laboratory examinations every 3 months for the first 2 years, every 6 months during the third to fifth years until patient death.

Cell culture and transfection. The human extrahepatic CC cell line QBC939 were purchased from a cell bank at the Chinese Academy of Sciences and grown in RPMI-1640 medium (Hyclone, Logan, UT, USA) supplemented with $10 \%$ fetal calf serum (Gibco, Grand Island, NY, USA). All cell lines were cultured at $37^{\circ} \mathrm{C}$ in a humidified atmosphere of $5 \% \mathrm{CO}_{2}$. Transfection reagent Lipofectamine 2000 was purchased from Invitrogen (St. Louis, MO, USA). For overexpression of FOXJ2, the full-length FOXJ2 cDNA was amplified and cloned into the pEGFP-N-3 expression vector (GeneChem, Shanghai, China). QBC939 cells were then transfected with a negative control vector or a FOXJ2 expressing plasmid using Lipofectamine 2000 according to the manufacturer's instructions.

Real-time quantitative PCR. Total RNA was extracted from tissues lysate using a TRIzol kit (Invitrogen, Carlsbad, CA, USA), and cDNA was subsequently synthesized from total RNA using an Omniscript RT kit (Qiagen, Valencia, CA, USA) according to the manufacturer's instructions. For detecting the mRNA level of FOXJ2, quantitative real-time RT-PCR was conducted on the Mastercycler ep realplex (Eppendorf $2 \mathrm{~S}$, Hamburg, Germany). A 25- $\mu$ l reaction mixture contained $1 \mu \mathrm{l}$ of cDNA from samples, $12.5 \mu \mathrm{l}$ of $2 \mathrm{X}$ Fast EvaGreen ${ }^{\mathrm{TM}} \mathrm{qPCR}$ Master Mix, $1 \mu 1$ primers (10 mM), and $10.5 \mu 1$ of RNase/ DNase-free water. PCR procedures: incubation at $96^{\circ} \mathrm{C}$ for $2 \mathrm{~min}, 40$ cycles at $96^{\circ} \mathrm{C}$ for $15 \mathrm{sec}$ and $60^{\circ} \mathrm{C}$ for $1 \mathrm{~min}$. The $\mathrm{Ct}$ value was defined as the cycle number at which the fluorescence intensity reached a certain threshold where amplification of each target gene was within the linear region of the reaction amplification curves. Relative expression level for each target gene was normalized by the Ct value of GAPDH (internal control) using the $2{ }^{\Delta \Delta \mathrm{Ct}}$ relative quantification method. The sequences of the primers for FOXJ2 were: FOXJ2 forward: 5'-TATGGTAGGGCATGAGGACAAC-3'; FOXJ2 reverse: 5'-GCAAACAATTAAAGGAGGACAAAC-3'. The glyceraldehyde-3' phosphate dehydrogenase (GAPDH) gene served as an internal control.

Western blot analysis. Since January 2013, fresh surgical specimens after surgical removal were collected and immediately frozen in liquid nitrogen until used for western blot analysis. The extrahepatic CC samples, including tumor and para-carcinoma normal tissues, as well as cell lines, were lysed in RIPA lysis buffer, and the lysates were harvested by centrifugation $(12,000 \mathrm{rpm})$ at $4^{\circ} \mathrm{C}$ for $30 \mathrm{~min}$. Approximately $50-\mathrm{mg}$ protein samples were then separated by electrophoresis in a $12 \%$ sodium dodecyl sulfate polyacrylamide gel and transferred onto a polyvinylidene fluoride membrane. After blocking the non-specific binding sites for 60 min with $5 \%$ non-fat milk, the membranes were incubated overnight at $4^{\circ} \mathrm{C}$ with a goat polyclonal antibody against FOXJ2 (Santa Cruz Biotechnology, USA at a 1:1,000 dilution). The membranes were then washed three times with TBST (Tris-buffered saline with Tween-20) 
for $10 \mathrm{~min}$ and probed with the horseradish peroxidase (HRP)conjugated donkey anti-goat IgG antibody (Immunology Consultants Laboratory, USA, at a 1:2,000 dilution) at $37^{\circ} \mathrm{C}$ for $1 \mathrm{~h}$. After three washes, the membranes were developed by an enhanced chemiluminescence system (Cell Signaling Technology, Danvers, MA, USA). The band intensity was measured by densitometry using Quantity One software (BioRad Laboratories, Inc., Hercules, CA, USA). The protein levels were normalized to glyceraldehyde-3-phosphate dehydrogenase (GAPDH).

Immunohistochemistry (IHC). The paraffin-embedded sections were deparaffinized with dimethylbenzene and rehydrated with raded alcohol solutions. After three washes in phosphate-buffered saline (PBS), the slides were boiled in antigen retrieval buffer containing $0.01 \mathrm{M}$ sodium citratehydrochloric acid ( $\mathrm{pH}$ 6.0) for $15 \mathrm{~min}$ in a microwave oven. After rinsing with PBS, the tissue sections were incubated with goat polyclonal anti-human FOXJ2 antibody (1:100, Santa Cruz Biotechnology, Inc., USA) and E-cadherin (diluted 1:1,000, Santa Cruz Biotechnology, Inc.), and then rinsed in 3\% peroxidase quenching solution (Invitrogen) to block endogenous peroxidase. The sections were then incubated with a donkey anti-goat second antibody conjugated horseradish peroxidase (1:5,000; Abcam, Cambridge, UK) at $4^{\circ} \mathrm{C}$ overnight. After washing in PBS, the visualization signal was developed with 3, 3'-diaminobenzidine (DAB) solution, and all of the slides were counterstained with hematoxylin. As negative controls, adjacent sections were processed as described above except that they were incubated overnight at $4^{\circ} \mathrm{C}$ in blocking solution without the primary antibody. The IHC results were scored by two experienced pathologists, who were blinded to clinical data. The total FOXJ2 immunostaining score was calculated as the sum of the percentage of positively stained tumor cells and the staining intensity and ranged from 0 to 9 . Briefly, the percentage of positive staining was scored as 0 (0-9\%, negative), 1 (10-25\%, sporadic), 2 (26-50\%, focal) or 3 (51-100\%, diffuse), and the intensity was scored as 0 (no staining), 1 (weak staining), 2 (moderate staining) or 3 (strong staining). The expression level of FOXJ2 was defined as follows: '-' (negative, score of 0), ' + ' (weakly positive, score of 1-3), '++' (positive, score of 4-6), ' +++ ' (strongly positive, score of 7-9). We defined strong FOXJ2 expression as a total score of $>3$, and weak FOXJ2 expression as a total score of $\leq 3$. As described elsewhere (22), we evaluated the intensity of E-cadherin staining on tumor cells, based on the staining of the control normal bile duct epithelium. E-cadherin was considered positive (high expression), if the staining intensity on tumor cells was the same as in normal bile duct epithelial cells. When the intensity of E-cadherin staining on tumor cells was weaker than the normal cells, E-cadherin was considered negative (low expression).

Cell proliferation assay. The tetrazolium-based cell viability (MTT) assay was performed to test cell proliferation. Cells transfected with the FOXJ2 plasmid or empty vector were seeded in a 96 -well plate at $1 \times 10^{3}$ cells/well containing $200 \mu 1$ DMEM supplemented with 10\% FBS. After 1, 2 and 3 days of incubation, $100 \mu \mathrm{l}$ of sterile MTT dye $(0.5 \mathrm{mg} / \mathrm{ml}$,
Sigma) was added to each well and cultured for another $4 \mathrm{~h}$. The supernatant was discarded and then $150 \mu 1$ of dimethyl sulphoxide (DMSO) (Sigma, St. Louis, MO, USA) was added to each well, the spectrophotometric absorbance was measured for each sample at $490 \mathrm{~nm}$, all the experiments were performed in triplicate and repeated 3 times, and the average was calculated.

Cell invasion and migration assays. The cell invasion capacity was determined using transwell chambers (Corning, Corning, NY, USA). The membrane filters were coated with Matrigel.

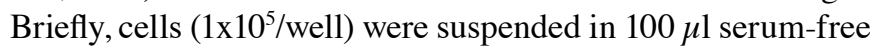
medium and then added to the upper chamber of the inserts, RPMI-1640 medium (Gibco) containing 10\% FBS (500 $\mu$ l) was added to the lower chamber as the chemotactic factor. After $48 \mathrm{~h}$ of incubation, the cells that had invaded through the Matrigel were visualized using $0.1 \%$ crystal violet staining. The numbers of migrated cells were calculated by counting five different views under the microscope. Independent experiments were repeated three times.

In addition, we examined migration using QBC939 cells that were transfected with either pEGFP-N-3-FOXJ2 or control vector. Transfected cells were cultured on 6-well plates. The confluent monolayers were scraped in a line across the slides with a sterile $20-\mu 1$ plastic pipette tip and incubated in serum-free medium for $48 \mathrm{~h}$. Plates were then imaged at 0,12 , 24 and $48 \mathrm{~h}$ with Olympus IX71 fluorescence microscope with a TH4-200 camera. Quantification was blinded, and performed by creating a longitudinal axis over the area of minimal density that corresponded to the site of wound formation. The average baseline wound area was centered over the axis and all the cells presented in that area were assumed to have migrated there. These cells were counted for data analysis.

Statistical analysis. All quantified data represented an average of at least triplicate samples. SPSS 17.0 (SPSS Inc, Chicago, IL, USA) was used for statistical analysis. Data are expressed as mean \pm SEM. The significance of the differences between values was determined using Student's t-test. $\chi^{2}$ test or Fischer's were used to identify differences between categorical variables. Survival curves for the patients were calculated using the Kaplan-Meier method and analyzed using the log-rank test. Prognostic factors were examined by univariate and multivariate analyses using a Cox proportional hazards model. Values of $\mathrm{P}<0.05$ were considered to indicate statistically significant results in all cases.

\section{Results}

Downregulated expression of FOXJ2 gene in extrahepatic CC and adjacent non-cancer normal bile duct tissues. In order to assess the role of FOXJ2 in extrahepatic CC, we performed real-time PCR to measure the expression of FOXJ2 mRNA in 21 freshly collected extrahepatic CC tissues and corresponding paracancer normal bile duct tissues. FOXJ2 protein was found to be markedly downregulated in 19 cases of extrahepatic CC compared with corresponding adjacent non-cancer normal bile duct tissues by western blotting ( $\mathrm{P}=0.029$, Fig. 1B and $\mathrm{C})$. In the 21 extrahepatic $\mathrm{CC}$ specimens, 9 cases had lymph node metastasis, and the other 12 cases were without metastasis. 

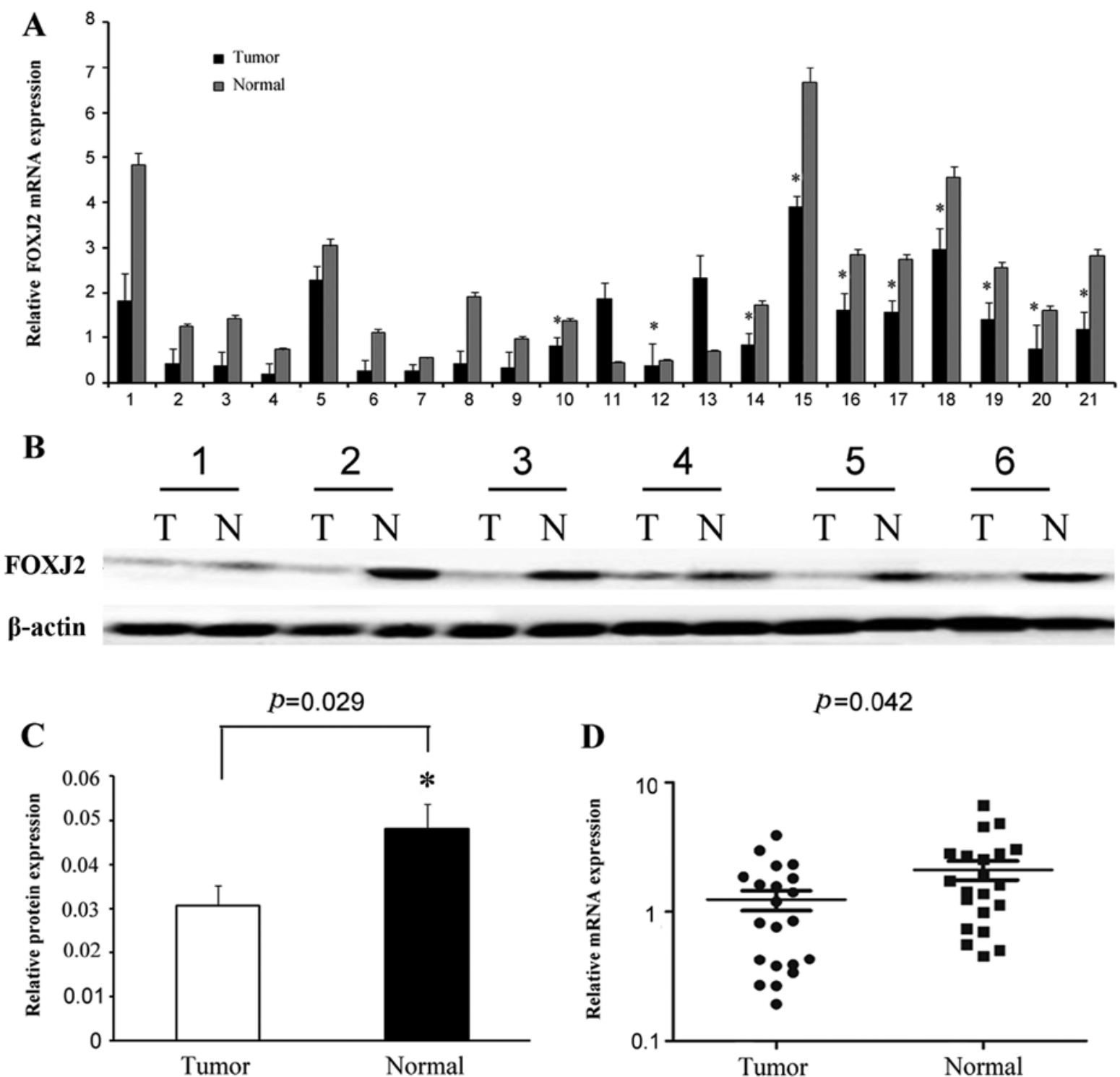

Figure 1. Expression of FOXJ2 in extrahepatic CC tissues. (A) The FOXJ2 mRNA expression in extrahepatic CC tissues compared to paired adjacent normal tissues was evaluated by real-time PCR. 1-9, extrahepatic CC with lymph node metastasis; 10-21, extrahepatic CCA without lymph node metastasis. The expression levels are normalized with GAPDH mRNA in each sample. The data are means \pm SEM ("P $<0.05$, compared with extrahepatic CC with lymph node metastasis). (B) Expression of FOXJ2 in six representative paired samples of extrahepatic CC tissues (T) and adjacent normal tissues (N). $\beta$-actin was used as a control for equal loading. (C) Western blot analysis shows that FOXJ2 protein expression was decreased in extrahepatic CC compared with adjacent normal tissues $(\mathrm{P}=0.029)$. The bar chart demonstrates the ratio of FOXJ2 protein to $\beta$-actin by densitometry. The data are means \pm SEM. (D) The average relative expression of mRNA level of FOXJ2 in extrahepatic $\mathrm{CC}$ tissues compared to paired adjacent normal tissues $(\mathrm{P}=0.042)$.

We also found that the mRNA level of FOXJ2 in metastatic extrahepatic CCs was significantly decreased compared with that in the extrahepatic CCs without lymph node metastasis $\left({ }^{*} \mathrm{P}<0.05\right.$, Fig. 1A). Compared with match paracancer normal tissues, extrahepatic CC tissues exhibited lower expression levels of FOXJ2 mRNA ( $\mathrm{P}=0.042$, Fig. 1D). We also measured the expression levels and subcellular localization of FOXJ2 in 63 paraffin-embedded extrahepatic CC samples by immunohistochemistry (Fig. 2). FOXJ2 protein showed low expression in $66.7 \%$ (42/63) of extrahepatic CC samples, while high expression in $33.3 \%$ (21/63) of extrahepatic CC samples, with staining mainly observed in the nucleus of the tumor cells.
The correlations between the expression of FOXJ2 and various clinicopathological characteristics. The relationship between clinicopathologic characteristics and FOXJ2 expression levels in individuals with extrahepatic CC were assessed by the $\chi^{2}$ analysis. We found no significant association between FOXJ2 expression levels and the patient age, sex, location, tumor size, differentiation, perineural invasion or tumor stage in the 63 extrahepatic CC cases. However, we observed that the expression level of FOXJ2 was positively correlated with lymphatic invasion $(\mathrm{P}=0.045)$, venous invasion $(\mathrm{P}=0.031)$, lymph node metastasis $(\mathrm{P}=0.012)$, TNM stage $(\mathrm{P}=0.006)$ and E-cadherin expression $(\mathrm{P}=0.011)$ in extrahepatic $\mathrm{CC}$ patients (Table I). 
Table I. Correlation between FOXJ2 expression and the clinicopathological characteristics of extrahepatic CC patients.

\begin{tabular}{|c|c|c|c|c|c|}
\hline \multirow[b]{2}{*}{ Factor } & \multirow[b]{2}{*}{ Cases $(n=63)$} & \multicolumn{4}{|c|}{ FOXJ2 expression } \\
\hline & & Low $(n=42)$ & High $(n=21)$ & P-value ${ }^{a}$ & $\chi^{2}$ \\
\hline Age & & & & 0.589 & 0.292 \\
\hline$\leq 60$ & 27 & 19 & 8 & & \\
\hline$>60$ & 36 & 23 & 13 & & \\
\hline Gender & & & & 0.202 & 1.625 \\
\hline Male & 38 & 23 & 15 & & \\
\hline Female & 25 & 19 & 6 & & \\
\hline Location & & & & 0.475 & 0.511 \\
\hline Hilar & 34 & 24 & 10 & & \\
\hline Distal & 29 & 18 & 11 & & \\
\hline Tumor size & & & & 0.280 & 1.167 \\
\hline$\leq 2 \mathrm{~cm}$ & 27 & 16 & 11 & & \\
\hline$>2 \mathrm{~cm}$ & 36 & 26 & 10 & & \\
\hline Differentiation & & & & 0.143 & 3.886 \\
\hline Well & 12 & 6 & 6 & & \\
\hline Moderate & 33 & 21 & 12 & & \\
\hline Poor & 18 & 15 & 3 & & \\
\hline Lymphatic invasion & & & & $0.045^{\mathrm{b}}$ & 4.012 \\
\hline- & 25 & 13 & 12 & & \\
\hline+ & 38 & 29 & 9 & & \\
\hline Venous invasion & & & & $0.031^{\mathrm{b}}$ & 4.667 \\
\hline- & 27 & 14 & 13 & & \\
\hline+ & 36 & 28 & 8 & & \\
\hline Perineural invasion & & & & 0.205 & 1.604 \\
\hline- & 26 & 15 & 11 & & \\
\hline+ & 37 & 27 & 10 & & \\
\hline Tumor stage & & & & 0.209 & 1.575 \\
\hline T1-2 & 35 & 21 & 14 & & \\
\hline T3-4 & 28 & 21 & 7 & & \\
\hline Lymph node metastasis & & & & $0.012^{\mathrm{b}}$ & 6.262 \\
\hline - & 34 & 18 & 16 & & \\
\hline+ & 29 & 16 & 5 & & \\
\hline TNM stage (UICC) & & & & $0.006^{\mathrm{b}}$ & 7.572 \\
\hline I-II & 39 & 21 & 18 & & \\
\hline III-IV & 24 & 21 & 3 & & \\
\hline E-cadherin expression & & & & $0.011^{\mathrm{b}}$ & 6.499 \\
\hline Low & 38 & 30 & 8 & & \\
\hline High & 25 & 12 & 13 & & \\
\hline
\end{tabular}

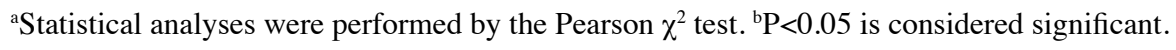

Expression of FOXJ2 and clinical outcome. The overall survival of patients with low FOXJ2 expression was significantly poorer than that of FOXJ2-high patients $(\mathrm{P}<0.001$, log-rank test (Fig. 3). Univariate Cox regression analyses showed that FOXJ2 expression was a prognostic factor for poor survival $(\mathrm{P}<0.001)$, differentiation, lymphatic invasion, 
Table II. Univariate and multivariate analysis of prognostic factors using Cox proportional hazards model.

\begin{tabular}{|c|c|c|c|c|c|c|}
\hline \multirow[b]{2}{*}{ Factor } & \multicolumn{3}{|c|}{ Univariate analysis } & \multicolumn{3}{|c|}{ Multivariate analysis } \\
\hline & HR & $95 \%$ CI & P-value & HR & $95 \%$ CI & P-value \\
\hline Age $(\leq 60 />60)$ & 0.628 & $0.368-1.070$ & 0.087 & - & - & - \\
\hline $\operatorname{Sex}(M / F)$ & 1.241 & $0.731-2.108$ & 0.423 & - & - & - \\
\hline Location (hilar/distal) & 0.884 & $0.524-1.491$ & 0.664 & - & - & - \\
\hline Tumor size $(\leq 2 \mathrm{~cm} />2 \mathrm{~cm})$ & 1.514 & $0.888-2.579$ & 0.127 & - & - & - \\
\hline Differentiation (well/mod/poor) & 2.136 & $1.040-4.390$ & $0.039^{\mathrm{a}}$ & 1.631 & $0.782-3.402$ & 0.192 \\
\hline Lymphatic invasion $(-/+)$ & 2.448 & $1.395-4.295$ & $0.002^{\mathrm{a}}$ & 1.621 & $0.909-2.893$ & 0.102 \\
\hline Venous invasion $(-/+)$ & 2.339 & $1.332-4.106$ & $0.003^{\mathrm{a}}$ & 1.545 & $0.876-2.752$ & 0.140 \\
\hline Perineural invasion (-/+) & 1.653 & $0.915-2.669$ & 0.102 & - & - & - \\
\hline Tumor stage (T1-2/ 3-4) & 2.778 & $1.615-4.779$ & $<0.001^{\mathrm{a}}$ & 2.008 & $1.149-3.509$ & $0.014^{\mathrm{a}}$ \\
\hline Lymph node metastasis $(-/+)$ & 3.121 & $1.807-5.393$ & $<0.001^{\mathrm{a}}$ & 2.161 & $1.226-3.809$ & $0.008^{\mathrm{a}}$ \\
\hline FOXJ2 expression (low/high) & 0.277 & $0.141-0.545$ & $<0.001^{\mathrm{a}}$ & 2.393 & $1.215-4.712$ & $0.012^{\mathrm{a}}$ \\
\hline
\end{tabular}

HR, hazard ratio; $\mathrm{CI}$, confidence interval; ${ }^{\mathrm{a}} \mathrm{P}<0.05$ was considered significant.

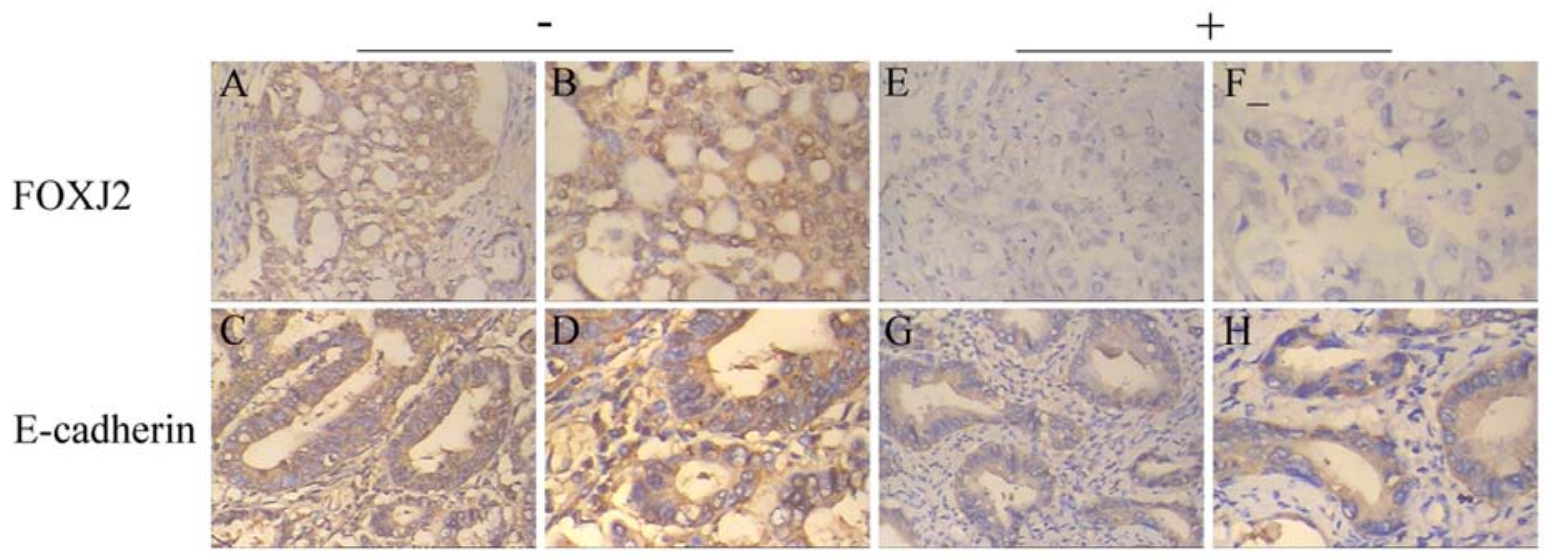

Figure 2. Immunohistochemical staining exhibits the detection of FOXJ2 and E-cadherin expression in paraffin-embedded extrahepatic CC tissues. '-' represents that lymph node metastasis is negative; '+' represents that lymph node metastasis is positive. (A-D) Tumor tissues with no metastasis showed high FOXJ2 and E-cadherin expression. (E-H) Tumor tissues with metastases showed low FOXJ2 and E-cadherin expression. Original magnification, x200 and x400.

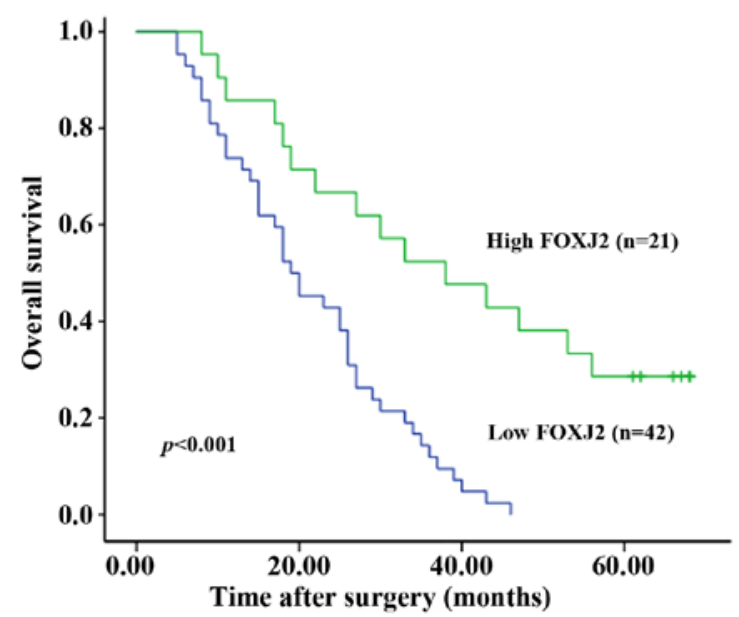

Figure 3. By comparison of overall survival cumulative Kaplan-Meier curves for patients grouped by immunohistochemical levels of FOXJ2, Kaplan-Meier curves for OS in extrahepatic CCA patients with low level and high level FOXJ2 expression $\left(\chi^{2}=15.918, \mathrm{P}<0.001\right)$. venous invasion, tumor stage and lymph node metastasis were also prognostic factors in the univariate analysis (Table II). Furthermore, a multivariate Cox regression analysis confirmed FOXJ2 expression $(\mathrm{P}=0.012)$, tumor stage and lymph node metastasis as independent predictors of the overall survival of extrahepatic CC patients (Table II).

Overexpression of FOXJ2 inhibits extrahepatic CC cell invasion, migration and proliferation in vitro. Because our above results indicated that FOXJ2 expression was reduced in extrahepatic CC and FOXJ2 might act as a tumor suppressor, we next explored the function of FOXJ2 in extrahepatic CC development. To evaluate the effects of FOXJ2 on cell invasion, the FOXJ2 overexpressing vector and the empty vector were respectively transfected into QBC939 cells. The cells were seeded in the chamber and their invasion ability was determined $48 \mathrm{~h}$ later. The results revealed that overexpression of FOXJ2 was associated with a significant reduction of 

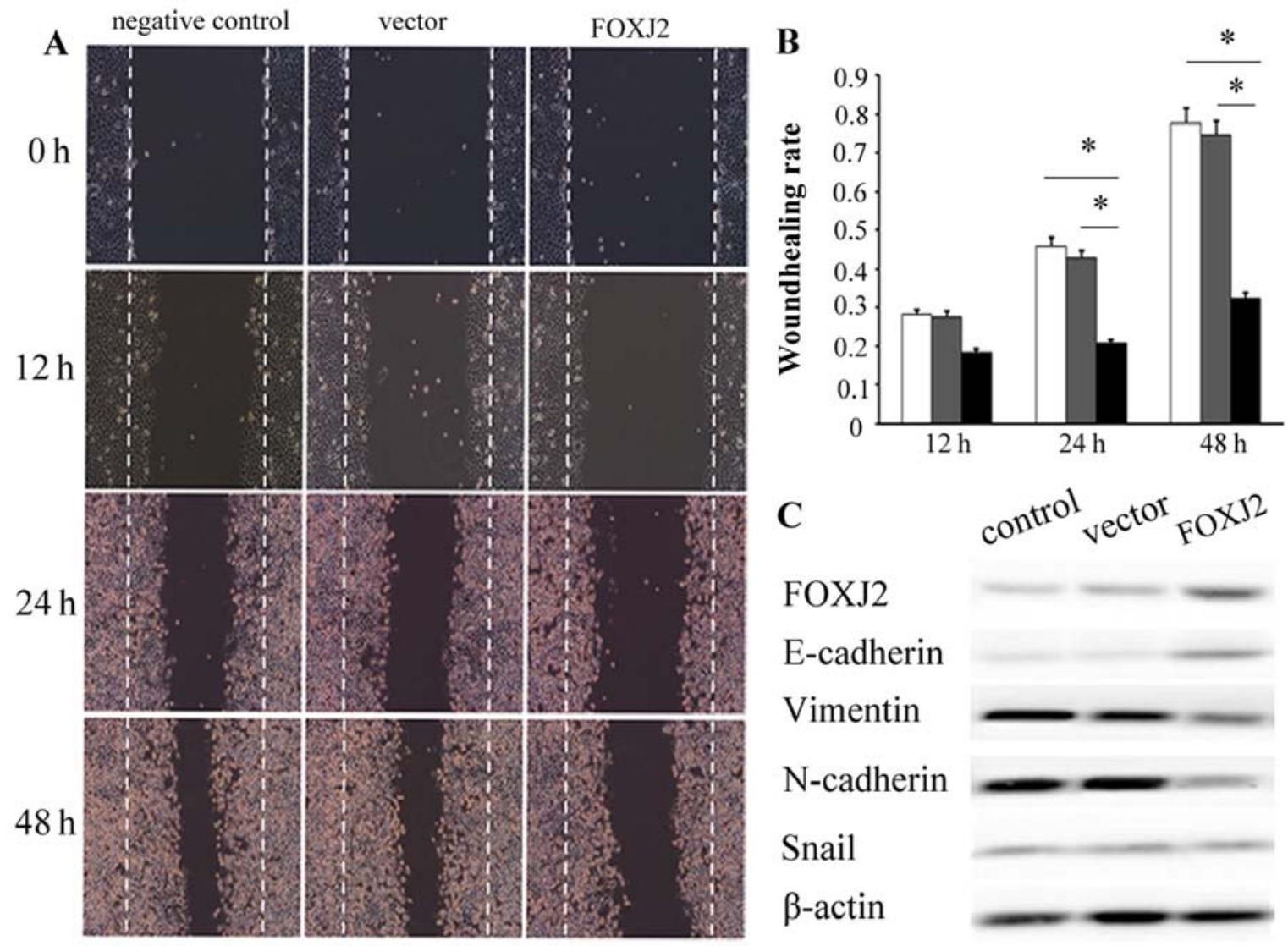

\section{E-cadherin \\ Vimentin}

N-cadherin

Snail

$\beta$-actin

Figure 4. (A and B) QBC939 cells were transfected with either pEGFP-N3-FOXJ2 (FOXJ2) or pEGFP-N3-vector (vector) for 12, 24 and $48 \mathrm{~h}$, respectively (" $\mathrm{P}<0.05$ compared to empty vector). (C) Western blot analysis showed that cells with exogenous expression of FOXJ2 expressed epithelial markers (E-cadherin) and showed loss of mesenchymal markers (vimentin and $\mathrm{N}$-cadherin). However, snail was not significantly changed. $\beta$-actin was used as a control.

A negative control

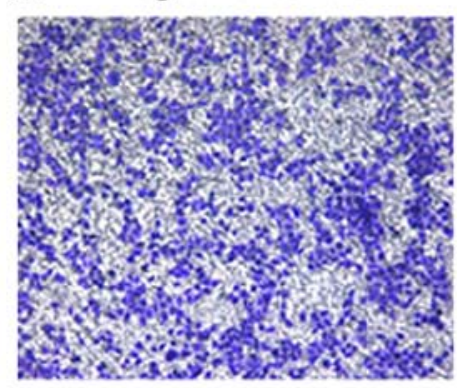

vector

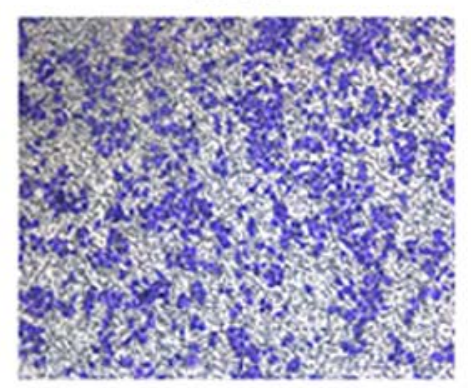

FOXJ2

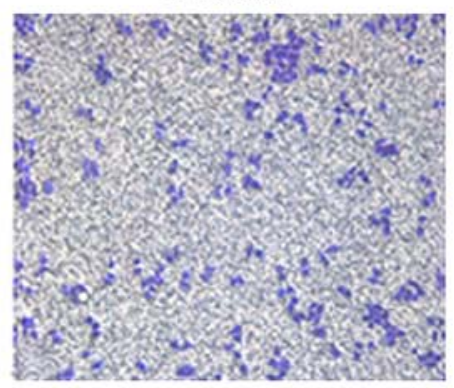

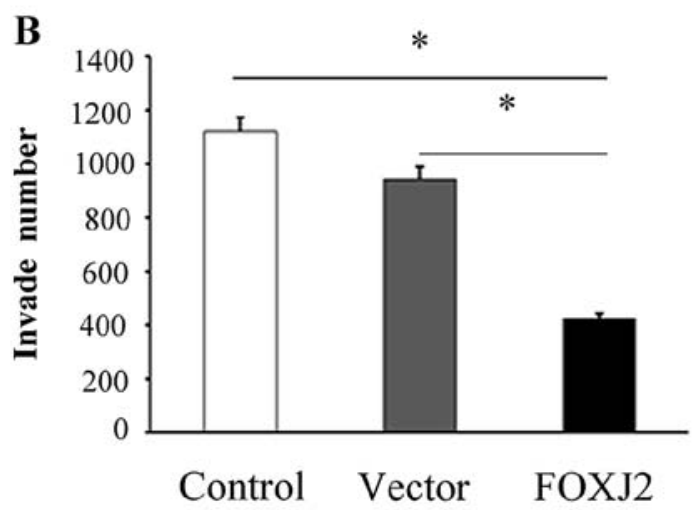

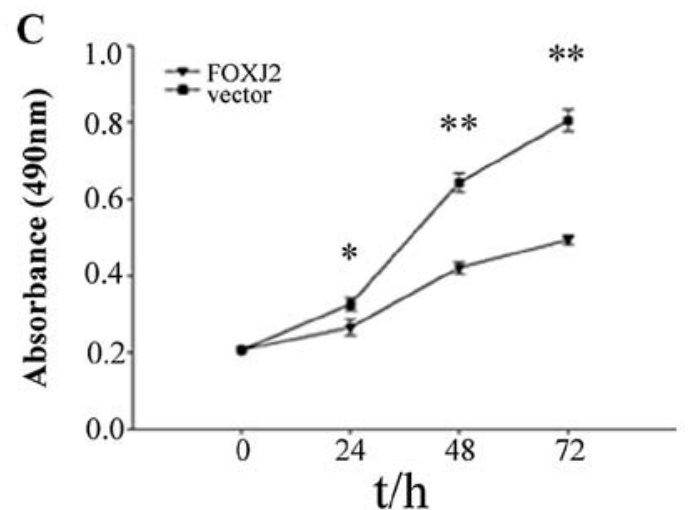

Figure 5. (A and B) Exogenous expression of FOXJ2 inhibited cell invasion in QBC939 cells as demonstrated by transwell assays ( $\mathrm{P}=0.039)$. Representative images of stained cells are shown with the original magnification of x100. (C) QBC939 cells transfected with pEGFP-N3-FOXJ2 (FOXJ2) had significantly reduced proliferation of cells compared with cells transfected with pEGFP-N3-vector (vector) $\left({ }^{*} \mathrm{P}<0.05,{ }^{* *} \mathrm{P}<0.01\right)$. Cell vitality was evaluated with the MTT assay using absorbance readings at $490 \mathrm{~nm}$. The values shown are the mean of three determinations. 
invasion compared to control empty vector extrahepatic $\mathrm{CC}$ cells (Fig. 5A and $\mathrm{B}, \mathrm{P}=0.039$ ), which showed that FOXJ2 significantly repressed the invasion of extrahepatic CC cells.

The above results were further confirmed by the wound healing assay. The overexpression of FOXJ2 significantly inhibited the migration of QBC939 cells at $48 \mathrm{~h}$ after transfection (Fig. 4A and B, $\mathrm{P}<0.05$ ). Moreover, the cell growth by MTT assay revealed that cell growth rate in FOXJ2-transfected extrahepatic CC cells were significantly lower than empty vector-transfected extrahepatic CC cells (Fig. 5C, $\mathrm{P}<0.05$ ). These data showed that the overexpression of FOXJ2 inhibited extrahepatic CC cell invasion, migration and proliferation in vitro.

To reveal how FOXJ2 regulates cell invasion and migration in QBC939 cells, we decided to study EMT because the cells acquire migrating potential and may invade the surrounding stroma and enter circulating blood (23). To determine whether EMT is involved in the effects of FOXJ2 on cell invasion and migration, change in expression level of EMT-related genes was measured by using western blotting, including E-cadherin, vimentin, N-cadherin and snail (Fig. 4C). The results imply that the changed expression of FOXJ2 was accompanied by the upregulation of epithelial marker E-cadherin and downregulation of mesenchymal marker vimentin and $\mathrm{N}$-cadherin, and the protein expression level of snail, a transcriptional regulator, was not significantly changed.

\section{Discussion}

Extrahepatic $\mathrm{CC}$ is an aggressive malignancy with dismal prognosis and characterized by early invasion, metastasis and postoperative recurrence. Therefore, understanding the main molecular mechanisms of this malignancy is the key for the development of novel and effective therapeutic strategies for extrahepatic CC. This is the first report on the clinicopathological significance of FOXJ2 expression in patients with extrahepatic CC. We found that the expression of FOXJ2 was significantly reduced at both mRNA and protein levels in extrahepatic CCs compared with paired paracancer normal bile duct tissues. We also found that extrahepatic CC patients with low expression of FOXJ2 showed shorter postoperative survival than high FOXJ2 expression patients. Therefore, it is proposed that downregulation of FOXJ2 may contribute to extrahepatic $\mathrm{CC}$ initiation and progression.

Lymph node metastasis frequently occurs in patients with extrahepatic CC. Recent studies have reported rates for lymph node metastasis of $24-47 \%$ for hilar CC, and $25-63 \%$ for distal CC $(5,6,24-31)$. In the present study, lymph node involvement was found in $42.8 \%$ of all patients. Our results showed that both the level of FOXJ2 mRNA and immunostaining rates in the extrahepatic CCs with lymph node metastasis were significantly decreased compared with those in extrahepatic CCs without lymph node metastasis. In addition, we showed that the low level of FOXJ2 expression has a propensity to be associated with lymphatic invasion, and lymph node metastasis in extrahepatic CC patients. We also found that the expression level of FOXJ2 was positively correlated with of E-cadherin. According to previous reports, lymph node metastasis and E-cadherin may serve as independent prognostic factors for extrahepatic CC $(26,28,30,32-34)$. By combining with the results of univariate and multivariate analyses, these data suggested that FOXJ2 may be a new prognostic marker for extrahepatic CC patients after surgical resection.

Epithelial-mesenchymal transition (EMT) is essential for phenotypic transition during embryogenesis and wound healing, and could also be reactivated during the malignant progression of numerous cancers (35). During EMT, tumor cells are expected to lose their epithelial phenotype and gradually acquire a mesenchymal phenotype. E-cadherin is a transmembrane glycoprotein which serves as the prime mediator of epithelial adhesion and also plays a critical role in suppression of tumor progression (35). Some research has shown that expression of EMT-related proteins is closely associated with tumor progression and a poor prognosis in extrahepatic CC $(34,36)$, suggesting that the EMT process may act as an important molecular event during the progression and metastasis of extrahepatic CC.

Immunostaining of individual EMT markers, such as E-cadherin, has shown that high expression of FOXJ2 is correlated with high E-cadherin, while low expression of FOXJ2 is correlated with low E-cadherin in the same tissue. Western blotting showed that exogenous FOXJ2 overexpression resulted in the increase of epithelial markers E-cadherin, and decrease of mesenchymal marker vimentin and $\mathrm{N}$-cadherin, whereas FOXJ2 overexpression had no effect on Snail expression. Thus, the above data indicate that the FOXJ2 protein is able to bind to E-cadherin promoters and transactivate their transcription, which suggest that FoxJ2 is involved in the regulation of cell adhesion events (37).

FOX family genes are implicated in carcinogenesis through gene amplification, retroviral integration, chromosomal translocation, and transcriptional regulation (38). FOXJ2 belongs to the human Fox family and was able to activate transcription (19). Furthermore, FOXJ2 appeared to be involved in positively regulating the progression of the cell cycle or contributing to tumorigenesis (39). Currently, several FOX subfamilies such as FOXA, FOXC, FOXM, FOXO, FOXP have been shown to play an important role in tumorigenesis and the progression of certain cancers (9). Besides, previous evidence suggests that FOXJ2 might actively participate in the metastatic process. In this study, we identified and functionally characterized FOXJ2 as an important role in extrahepatic CC progression. Whereas, our data, obtained by modulating FOXJ2 expression, establish a role for FOXJ2 in modulating the biological properties of extrahepatic $\mathrm{CC}$ cells, including proliferation, migration and aggressiveness in vitro. Overexpression of FOXJ2 resulted in suppression of cell migration and invasion, which suggests a potential role for FOXJ2 in the regulation of tumor cell migration, invasion, in line with previous studies (21). Considering cell proliferation function of FOXJ2 identified in spinal cord injury, FOXJ2 expression was increased predominantly in astrocytes, which highly expressed proliferating cell nuclear antigen, a marker for proliferating cells (40), we suggest that exogenous FOXJ2 resulted in arrest of extrahepatic CC cell proliferation in this study.

Thus, based on previous studies and the present study, we suggest that overexpression of FOXJ2 contributed to extrahepatic CC initiation and progression through promoting the migration and invasion of extrahepatic $\mathrm{CC}$ cells, and possibly functions as a tumor suppressor gene, which is consist with in 
an earlier study (21). Therefore, the data in this study suggest reasons to believe that FOXJ2 could be a new therapeutic target for improving the treatment efficiency of extrahepatic cholangiocarcinoma.

In conclusion, we provide compelling evidence that overexpression of FOXJ2 leads to suppressed cell growth, migration and invasion in extrahepatic $\mathrm{CC}$ cells. FOXJ2 expression may be a therapeutic target, or useful to guide therapy of extrahepatic CC patients. However, the complex molecular mechanisms of FOXJ2 contributing to extrahepatic $\mathrm{CC}$ require further investigation.

\section{Acknowledgements}

This study was supported by Medical innovation team and talents of Jiangsu province (LJ201134).

\section{References}

1. Augustine MM and Fong Y: Epidemiology and risk factors of biliary tract and primary liver tumors. Surg Oncol Clin North Am 23: 171-188, 2014

2. Shaib Y and El-Serag HB: The epidemiology of cholangiocarcinoma. Semin Liver Dis 24: 115-125, 2004.

3. van der Gaag NA, Kloek JJ, de Bakker JK, Musters B, Geskus RB, Busch OR, Bosma A, Gouma DJ and van Gulik TM: Survival analysis and prognostic nomogram for patients undergoing resection of extrahepatic cholangiocarcinoma. Ann Oncol 23: 2642-2649, 2012.

4. Kaira K, Sunose Y, Ohshima Y, Ishioka NS, Arakawa K, Ogawa T, Sunaga N, Shimizu K, Tominaga H, Oriuchi N, et al: Clinical significance of L-type amino acid transporter 1 expression as a prognostic marker and potential of new targeting therapy in biliary tract cancer. BMC Cancer 13: 482, 2013.

5. Hirano S, Kondo S, Tanaka E, Shichinohe T, Tsuchikawa T, Kato K, Matsumoto J and Kawasaki R: Outcome of surgical treatment of hilar cholangiocarcinoma: A special reference to postoperative morbidity and mortality. J Hepatobiliary Pancreat Sci 17: 455-462, 2010.

6. Sakamoto Y,Kosuge T, Shimada K, Sano T, Ojima H, Yamamoto J, Yamasaki S, Takayama T and Makuuchi M: Prognostic factors of surgical resection in middle and distal bile duct cancer: An analysis of 55 patients concerning the significance of ductal and radial margins. Surgery 137: 396-402, 2005.

7. Kaufmann E and Knöchel W: Five years on the wings of fork head. Mech Dev 57: 3-20, 1996.

8. Lehmann OJ, Sowden JC, Carlsson P,Jordan T and Bhattacharya SS: Fox's in development and disease. Trends Genet 19: 339-344, 2003.

9. Myatt SS and Lam EW: The emerging roles of forkhead box (Fox) proteins in cancer. Nat Rev Cancer 7: 847-859, 2007.

10. Benayoun BA, Caburet S and Veitia RA: Forkhead transcription factors: Key players in health and disease. Trends Genet 27: 224-232, 2011.

11. Banham AH, Connors JM, Brown PJ, Cordell JL, Ott G, Sreenivasan G, Farinha P, Horsman DE and Gascoyne RD: Expression of the foxp1 transcription factor is strongly associated with inferior survival in patients with diffuse large B-cell lymphoma. Clin Cancer Res 11: 1065-1072, 2005.

12. Schuster MB and Porse BT: C/EBPalpha: A tumour suppressor in multiple tissues? Biochim Biophys Acta 1766: 88-103, 2006.

13. Barrans SL, Fenton JA, Banham A, Owen RG and Jack AS: Strong expression of FOXP1 identifies a distinct subset of diffuse large B-cell lymphoma (DLBCL) patients with poor outcome. Blood 104: 2933-2935, 2004

14. Banham AH, Beasley N, Campo E, Fernandez PL, Fidler C, Gatter K, Jones M, Mason DY, Prime JE, Trougouboff P, et al: The FOXP1 winged helix transcription factor is a novel candidate tumor suppressor gene on chromosome 3p. Cancer Res 61: 8820-8829, 2001.

15. Lin L, Miller CT, Contreras JI, Prescott MS, Dagenais SL, Wu R, Yee J, Orringer MB, Misek DE, Hanash SM, et al: The hepatocyte nuclear factor 3 alpha gene, HNF3alpha (FOXA1), on chromosome band $14 \mathrm{q} 13$ is amplified and overexpressed in esophageal and lung adenocarcinomas. Cancer Res 62: 5273-5279, 2002.
16. Chen HW, Huang XD, Li HC, He S, Ni RZ, Chen CH, Peng C, Wu G, Wang GH, Wang YY, et al: Expression of FOXJ1 in hepatocellular carcinoma: Correlation with patients' prognosis and tumor cell proliferation. Mol Carcinog 52: 647-659, 2013.

17. Lee JS, Chu IS, Heo J, Calvisi DF, Sun Z, Roskams T, Durnez A, Demetris AJ and Thorgeirsson SS: Classification and prediction of survival in hepatocellular carcinoma by gene expression profiling. Hepatology 40: 667-676, 2004.

18. Obama K, Ura K, Li M, Katagiri T, Tsunoda T, Nomura A, Satoh S, Nakamura Y and Furukawa Y: Genome-wide analysis of gene expression in human intrahepatic cholangiocarcinoma. Hepatology 41: 1339-1348, 2005.

19. Pérez-Sánchez C, Arias-de-la-Fuente C, Gómez-Ferrería MA, Granadino B and Rey-Campos J: FHX.L and FHX.S, two isoforms of the human fork-head factor FHX (FOXJ2) with differential activity. J Mol Biol 301: 795-806, 2000.

20. Pérez-Sánchez C, Gómez-Ferrería MA, de La Fuente CA, Granadino B, Velasco G, Esteban-Gamboa A and Rey-Campos J: FHX, a novel fork head factor with a dual DNA binding specificity. J Biol Chem 275: 12909-12916, 2000.

21. Wang Y, Yang S, Ni Q, He S, Zhao Y, Yuan Q, Li C, Chen H, Zhang L, Zou L, et al: Overexpression of forkhead box J2 can decrease the migration of breast cancer cells. J Cell Biochem 113: 2729-2737, 2012

22. Shiozaki H, Tahara H, Oka H, Miyata M, Kobayashi K, Tamura S, Iihara K, Doki Y, Hirano S, Takeichi M, et al: Expression of immunoreactive E-cadherin adhesion molecules in human cancers. Am J Pathol 139: 17-23, 1991.

23. Thiery JP, Acloque H, Huang RY and Nieto MA: Epithelialmesenchymal transitions in development and disease. Cell 139: 871-890, 2009.

24. Murakami Y, Uemura K, Sudo T, Hayashidani Y, Hashimoto Y, Nakamura H, Nakashima A and Sueda T: Gemcitabine-based adjuvant chemotherapy improves survival after aggressive surgery for hilar cholangiocarcinoma. J Gastrointest Surg 13: 1470-1479, 2009

25. Igami T, Nishio H, Ebata T, Yokoyama Y, Sugawara G, Nimura Y and Nagino M: Surgical treatment of hilar cholangiocarcinoma in the 'new era': The Nagoya University experience. J Hepatobiliary Pancreat Sci 17: 449-454, 2010.

26. Lee SG, Song GW, Hwang S, Ha TY, Moon DB, Jung DH, Kim KH, Ahn CS, Kim MH, Lee SK, et al: Surgical treatment of hilar cholangiocarcinoma in the new era: The Asan experience. J Hepatobiliary Pancreat Sci 17: 476-489, 2010

27. Unno M, Katayose Y, Rikiyama T, Yoshida H, Yamamoto K, Morikawa T, Hayashi H, Motoi F and Egawa S: Major hepatectomy for perihilar cholangiocarcinoma. J Hepatobiliary Pancreat Sci 17: 463-469, 2010

28. Murakami Y, Uemura K, Hayashidani Y, Sudo T, Hashimoto Y, Ohge $\mathrm{H}$ and Sueda T: Prognostic significance of lymph node metastasis and surgical margin status for distal cholangiocarcinoma. J Surg Oncol 95: 207-212, 2007.

29. Ebata T, Nagino M, Nishio H, Igami T, Yokoyama $Y$ and Nimura Y: Pancreatic and duodenal invasion in distal bile duct cancer: Paradox in the tumor classification of the American Joint Committee on Cancer. World J Surg 31: 2008-2015, 2007.

30. Woo SM, Ryu JK, Lee SH, Yoo JW, Park JK, Kim YT, Jang JY, Kim SW, Kang GH and Yoon YB: Recurrence and prognostic factors of ampullary carcinoma after radical resection: Comparison with distal extrahepatic cholangiocarcinoma. Ann Surg Oncol 14: 3195-3201, 2007.

31. Hong SM, Pawlik TM, Cho H, Aggarwal B, Goggins M, Hruban RH and Anders RA: Depth of tumor invasion better predicts prognosis than the current American Joint Committee on Cancer T classification for distal bile duct carcinoma. Surgery 146: 250-257, 2009.

32. Murakami Y, Uemura K, Sudo T, Hashimoto Y, Nakashima A, Kondo N, Sakabe R, Ohge H and Sueda T: Prognostic factors after surgical resection for intrahepatic, hilar, and distal cholangiocarcinoma. Ann Surg Oncol 18: 651-658, 2011.

33. Li Q, Wang JM, Liu C, Xiao BL, Lu JX and Zou SQ: Correlation of aPKC-iota and E-cadherin expression with invasion and prognosis of cholangiocarcinoma. Hepatobiliary Pancreat Dis Int 7: 70-75, 2008.

34. Nitta T, Mitsuhashi T, Hatanaka Y, Miyamoto M, Oba K, Tsuchikawa T, Suzuki Y, Hatanaka KC, Hirano S and Matsuno Y: Prognostic significance of epithelial-mesenchymal transition-related markers in extrahepatic cholangiocarcinoma: Comprehensive immunohistochemical study using a tissue microarray. Br J Cancer 111: 1363-1372, 2014. 
35. Thiery JP: Epithelial-mesenchymal transitions in tumour progression. Nat Rev Cancer 2: 442-454, 2002.

36. Zhang KJ, Wang DS, Zhang SY, Jiao XL, Li CW, Wang XS, $\mathrm{Yu} \mathrm{QC}$ and Cui HN: The E-cadherin repressor slug and progression of human extrahepatic hilar cholangiocarcinoma. J Exp Clin Cancer Res 29: 88, 2010.

37. Martín-de-Lara F, Sánchez-Aparicio P, Arias de la Fuente C and Rey-Campos J: Biological effects of Fox $\mathrm{J} 2$ over-expression. Transgenic Res 17: 1131-1141, 2008.
38. Katoh $\mathrm{M}$ and Katoh M: Human FOX gene family (Review). Int J Oncol 25: 1495-1500, 2004.

39. Kehn K, Berro R, Alhaj A, Bottazzi ME, Yeh WI, Klase Z, Van Duyne R, Fu S and Kashanchi F: Functional consequences of cyclin D1/BRCA1 interaction in breast cancer cells. Oncogene 26: 5060-5069, 2007.

40. Chen X, Cao X, Tao G, Cao Z, Wang S, Zhou F, Xie W, Zhao P, Zhang Z and Cui Z: Foxj2 expression in rat spinal cord after injury and its role in inflammation. J Mol Neurosci 47: 158-165, 2012. 